\title{
Interventions provided in the acute phase for mild traumatic brain injury: a systematic review
}

Jocelyn Gravel ${ }^{1,6^{*}}$, Antonio D'Angelo ${ }^{1}$, Benoit Carrière ${ }^{1}$, Louis Crevier ${ }^{2}$, Miriam H Beauchamp ${ }^{3,5}$, Jean-Marc Chauny ${ }^{4}$, Maggy Wassef ${ }^{5}$ and Nils Chaillet ${ }^{5}$

\begin{abstract}
Background: Most patients who sustain mild traumatic brain injury (mTBI) have persistent symptoms at 1 week and 1 month after injury. This systematic review investigated the effectiveness of interventions initiated in acute settings for patients who experience mTBI.

Methods: We performed a systematic review of all randomized clinical trials evaluating any intervention initiated in an acute setting for patients experiencing acute mTBI. All possible outcomes were included. The primary sources of identification were MEDLINE, Embase, PsycINFO, CINAHL, and the Cochrane Central register of Controlled Trials, from 1980 to August 2012. Hand searching of proceedings from five meetings related to mTBI was also performed. Study selection was conducted by two co-authors, and data abstraction was completed by a research assistant specialized in conducting systematic reviews. Study quality was evaluated using Cochrane's Risk of Bias assessment tool.

Results: From a potential 15,156 studies, 1,268 abstracts were evaluated and 120 articles were read completely. Of these, 15 studies fulfilled the inclusion/exclusion criteria. One study evaluated a pharmacological intervention, two evaluated activity restriction, one evaluated head computed tomography scan versus admission, four evaluated information interventions, and seven evaluated different follow-up interventions. Use of different outcome measures limited the possibilities for analysis. However, a meta-analysis of three studies evaluating various follow-up strategies versus routine follow-up or no follow-up failed to show any effect on three outcomes at 6 to 12 months post-trauma. In addition, a meta-analysis of two studies found no effect of an information intervention on headache at 3 months post-injury.
\end{abstract}

Conclusions: There is a paucity of well-designed clinical studies for patients who sustain mTBI. The large variability in outcomes measured in studies limits comparison between them.

Keywords: mTBI, Traumatic brain injury, Head concussion, Systematic review, Treatment

\section{Background}

Mild traumatic brain injury (mTBI) is defined as the presence of head trauma, a Glasgow Coma Scale (GCS) score of 13 to 15 , and at least one of the following four criteria: any period of loss of consciousness; any loss of memory for events immediately before or after the accident; any alteration in mental state at the time of the

\footnotetext{
* Correspondence: graveljocelyn@hotmail.com

'Département de Pédiatrie, CHU Sainte-Justine, Université de Montréal, Montréal, Canada

${ }^{6}$ Section d'Urgence, Département de Pédiatrie, CHU Sainte-Justine, 3175

Chemin Côte Sainte-Catherine, Montréal QC H3T 1C5, Canada

Full list of author information is available at the end of the article
}

accident (for example, feeling dazed); or focal neurological deficit(s) that may or may not be transient $[1,2]$.

The WHO estimates that the incidence of mTBI is of 600 cases per 100,000 adults in USA [3-5], with higher incidence for young adults and athletes [3,6-11]. For children, the WHO reports incidences varying from 50 to 100 cases per 100,000 children-years depending on age $[3,12-14]$. Although most studies suggest that the long-term evolution of mTBI is excellent, with complete resolution of symptoms in 3 months [15-17], 55 to $90 \%$ of patients who sustain mTBI experience post-concussion symptoms during the week following the accident $[15,18,19]$. The nature of these symptoms can be cognitive

\section{Biomed Central}

(c) 2013 Gravel et al.; licensee BioMed Central Ltd. This is an Open Access article distributed under the terms of the Creative Commons Attribution License (http://creativecommons.org/licenses/by/2.0), which permits unrestricted use, distribution, and reproduction in any medium, provided the original work is properly cited. 
(memory loss, attention deficit), somatic (headache, fatigue, nausea), or psychological (depression, irritability).

Most patients requiring medical resources secondary to mTBI are initially evaluated at the emergency department (ED). Guidelines pertaining to the management of mTBI generally recommend activity restriction and/or treatment of symptoms [20-23]. Few studies have evaluated potential interventions initiated in the ED for patients who sustain mTBI. A systematic review by the WHO was conducted to evaluate prevalence, outcome, and potential treatment for mTBI [24,25]. Only 16 studies describing potential treatments for mTBI were included. The main limitations of that review were that it included articles published only until the year 2000, and it did not evaluate the effects of interventions on outcomes at 1 week and 1 month. The objective of the current study was to identify all clinical trials of interventions that could be initiated in an acute setting for patients who sustain mTBI.

\section{Methods}

\section{Design}

This was a systematic review of the literature to identify randomized clinical trials evaluating any intervention in the acute phase of trauma versus any comparator or placebo for patients with mTBI.

\section{Data source and identification of studies}

A database search strategy was formulated by one of the authors (MW) who has experience in conducting systematic reviews. A literature search was performed to identify clinical trials and randomized clinical trials including patients with mTBI (GCS score between 13 and 15) seeking acute treatment, and comparing any intervention in the acute phase (first week) of trauma versus any comparator or placebo. Main outcomes measured were somatic post-concussion symptoms (headaches, dizziness, vision, fatigue, irritability, or sleep problems). Other symptoms also considered were cognitive symptoms (memory, attention, concentration, cognition, and language), psychological or emotional symptoms (anxiety, depression, or irritability), autonomy, return to activity (work, school or sport), physical disability, hospital or emergency room readmission, and any side effects of the intervention.

The literature search was conducted via OvidSP in the following electronic databases: Embase, MEDLINE, EBM Reviews, ACP Journal Club, Cochrane Central Register of Controlled Trials, PsychINFO and CinHAL. A Boolean search was constructed using the following MESH terms: 'head, brain, cerebral, craniocerebral' combined with any of the following MESH terms: 'trauma, injury, concussion or post concussion' and the following MESH term: 'clinical trial'. Appropriate MESH words were searched and adapted to each database. The search was limited to human subjects and articles published between 1980 and current (August 2012) with no language limitation.

An additional search was conducted in Google Scholar and PubMed, in the references from relevant reviews and clinical trials, and by using authors' names and searching in similar studies to identify potential additional clinical trials. A hand search of conference proceedings of the International Brain Injury Association, the International Neuropsychological Society, the Society of the American Association of Neurological Surgeon, the Society of Academic Emergency Medicine, and the Canadian Association of Emergency Physicians was also conducted for the years 2010, 2011, and 2012.

Inclusion criteria were: trials including patients, without age restriction, seeking acute treatment after a head trauma, with a GCS score between 13 and 15, and one of the following symptoms:

- Temporary loss of consciousness (less than 30 minutes).

- Amnesia of less than 24 hours.

- Altered state of consciousness.

- Transient focal neurological deficit.

All interventions were eligible. These included, but were not limited to, medications, psychological therapy, patient education (including brochure, pamphlets or meeting with a health professional), activity restriction, hospitalization, or bed rest, and follow-up in a specialized clinic.

Exclusion criteria were: all trials concerning only moderate to severe cases of TBI (GCS <13); clinical trials where time between the trauma and the intervention was more than 1 week; trials concerning only subgroups of patients (for example, patients with insomnia); trials with diagnoses or outcomes relevant only for moderate or severe TBI (for example, death); trials where the extraction of data regarding $\mathrm{mTBI}$ was not possible; and studies performed on animals or cadavers.

\section{Study selection}

Three authors (BC, AD, and JG) independently screened in duplicate all titles and abstracts identified by the search. The full manuscripts of all studies selected by at least one reviewer were then evaluated independently and discussed by two authors (BC and JG) to identify the clinical trials to be included. Finally, the full manuscripts of the clinical trials were reviewed independently in accordance with the inclusion and exclusion criteria, and listed to select the final studies included in this review. During these three steps, discrepancies were discussed and resolved by consensus between the authors, otherwise, a third author was consulted to reach an agreement. 


\section{Assessment of risk of bias in included studies}

Two authors (JG and MW) assessed the minimum inclusion criteria for randomized clinical trials described in the Cochrane Effective Practice and Organization of Care (EPOC) review group [26]. To be included, a study had to use a proper randomization tool to assign the patient prospectively, have objective measurement of performance, and have relevant and interpretable data presented or obtained. Two authors (JG and MW) independently assessed each study using the Risk of Bias tool for randomized clinical trials described in the method guide for comparative effectiveness [27]. The tool evaluated the following risks of bias: selection (sequence allocation, concealment, and confounding, analysis of patients in the group to which they were randomized), performance (concurrent intervention and fidelity to protocol), attrition, detection (length of follow-up, blinding of assessor, and validity of measurement of intervention, outcomes and confounders), and reporting bias. Each item received a rating of 'no' if judged to be at high risk of bias, a rating of 'yes' if judged to have a low risk of bias, and 'unclear' when the evaluator could not conclude the risk of bias. Any discordance between the authors was discussed and resolved by consensus. Each study was rated as having a low, unclear, or potential risk of bias.

\section{Data extraction and management}

Two authors (JG and MW) independently extracted data from each study using a standardized data-extraction checklist. The database included: demographic data of the study; general information (author, year of publication, year of enrollment in the study, country of the study, setting, study design, randomization tool used, and duration of the follow-up); patient information (number of patients, age, number of patients who dropped out, inclusion and exclusion criteria, and diagnosis of mTBI on admission); GCS score; intervention and comparator description; category and number of patients in each group and outcome description; category and measurement tools; and results of outcome of interest in detail including somatic, cognitive, emotional and 'return to activities' data. In cases of missing data or lack of transparency, authors were contacted for clarification. In the absence of a response, the data were considered unattainable. Discordances between reviewers were resolved by consensus. Data were then entered into the Cochrane Review Manager software (RevMan5, 2008) and checked for accuracy.

\section{Analysis}

The large heterogeneity in interventions, outcomes measured, and timing of assessment reported in the studies that met our inclusion criteria limited meta-analytic possibilities. A priori, it was decided to conduct a meta-analysis if two or more studies fulfilled the following three criteria: 1) they evaluated a similar intervention; 2) they measured the same outcome; 3) they measured the outcome during the same time frame. Based on the characteristics of the studies identified, two meta-analyses were performed to assess the mid-term effects (1 to 3 months) of information given during the visit to the ED versus routine care, and longterm effects (6 to 12 months) of phone or clinical follow-up versus routine care. Dichotomous data were meta-analyzed, using odds ratios (OR) with 95\% CI as measures of effect size, or Peto OR if the number of events in a group was equal to 0 . Inter-study variation was incorporated with the assumption of a random effects model for the treatment effect using the DerSimonian and Laird method and the inverse variance method for dichotomous data when heterogeneity between trials was significant or was greater than 50\% [26]. In all randomized clinical trials, outcomes were directly compared between the control and the intervention group on an intention-to-treat basis (ITT). The Q and $\mathrm{I}^{2}$ tests were used for addressing heterogeneity [26]. If significant heterogeneity was detected, subgroup analyses were carried out by type of study intervention.

\section{Ethics approval}

Because the study did not involve recruitment or assessment of any patients, no research ethics board evaluation was necessary.

\section{Results}

After removing duplicates, the literature search initially identified 15,156 potential studies (see Figure 1). Most of these were excluded based on their title because they were not related to mTBI. A total of 1,268 abstracts were evaluated, and 1,163 were excluded because they did not meet our inclusion/exclusion criteria. The full manuscripts of 105 articles were evaluated in addition to 15 articles identified through the bibliographies. Of these, 17 articles describing 15 distinct studies fulfilled the inclusion/exclusion criteria. A hand search of the five conference proceedings failed to identify any other potentially pertinent study.

The characteristics of the included studies are described in Table 1. Only one study evaluated a pharmacological intervention [28]. Four studies evaluated standardized information sessions provided in the acute setting with or without an information booklet [29-32]. Seven studies evaluated follow-up interventions compared with no follow-up or routine follow-up [33-39]. Two studies evaluated activity restriction (full bed rest for 6 days [40] and hospital admission for 24 hours [19]). Finally, one study evaluated the long-term outcome of performing a computed tomography (CT) scan of the head versus admission for patients with mTBI [41]. The size of the studies ranged from 17 to 2,602 participants, with a median of 262 participants. Patients of all ages were included. 


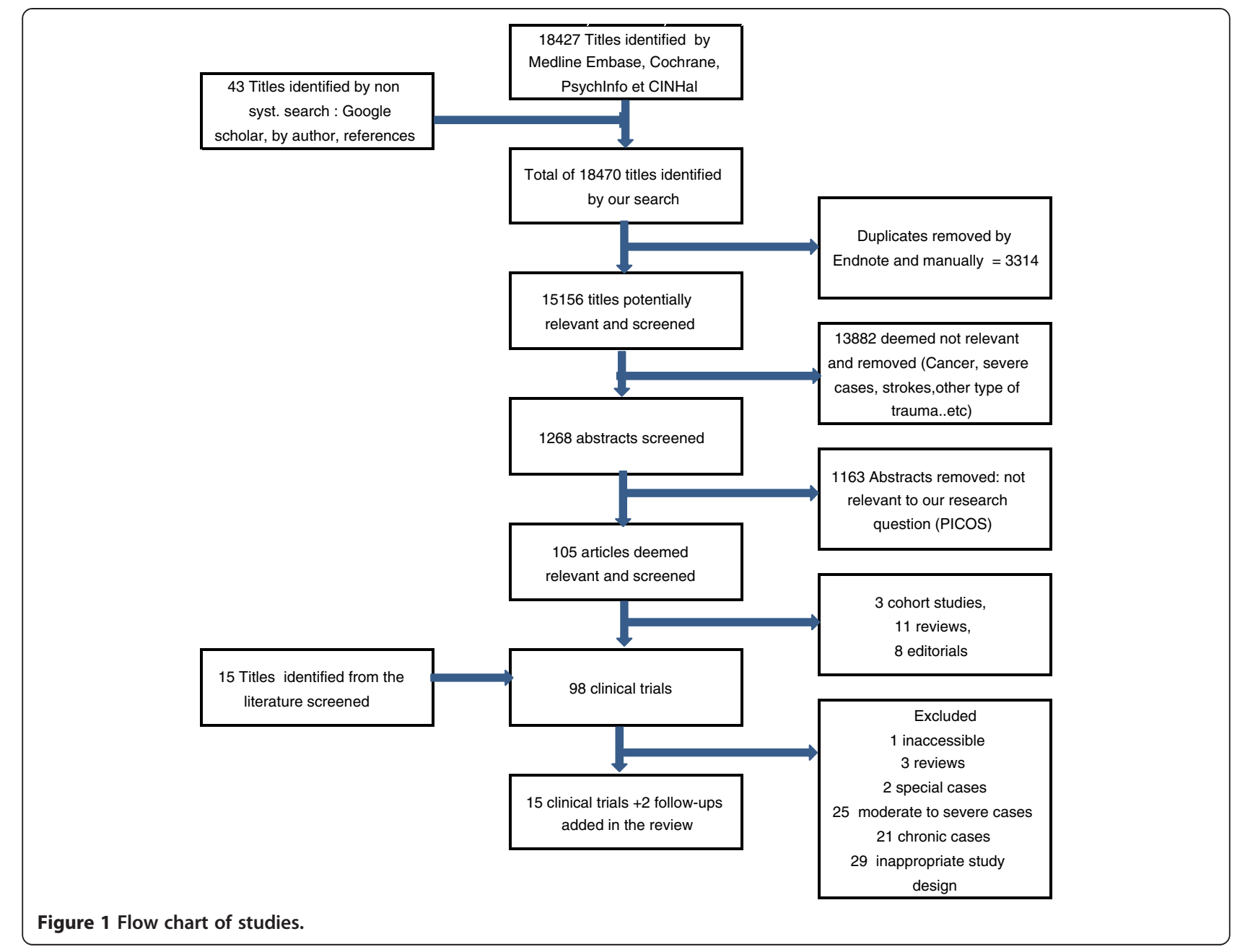

Three studies involved only children, one study involved children and adults, and eleven studies involved only adults or patients older than 15 years. Multiple outcomes were measured at intervals varying between 3 days and 10 years post-trauma.

Of the 15 studies, 5 presented a potential risk of bias. The main reasons for potential biases were related to the randomization sequence generation and the inadequate concealment of allocation of participants (Figure 2). The risk of bias was unclear for six studies. Most of these had unclear information about multiple components of the Risk of Bias tool. The most problematic components were sequence allocation and concealment.

\section{Interventions}

The only pharmacological study reported that, compared with placebo, nasal administration of $10 \mu \mathrm{g}$ bid of 1 desamino-8-d-arginine-vasopressin (DDAVP) for 5 days was associated with better performance on two memory tests on the third day of treatment for adults who sustained mTBI [28]. The main limitations of the study related to the small sample size $(n=17)$, the small statistical effect, and the fact that the intervention had no influence on four other cognitive outcomes.

Four studies evaluated a standardized information intervention provided in the ED. Three studies [29-31] reported that the intervention was not more effective than usual care in decreasing post-concussion symptoms, while one study [32] reported that meeting with a specialized therapist and the provision of a 10-page information booklet decreased post-concussion symptoms among adults admitted for mTBI. One study suggested that standardized information and reassurance provided at the ED was associated with faster return to work and social activities [30]. The baseline characteristics of these studies allowed us to conduct a meta-analysis using two studies $[29,30]$ that reported outcomes on individual signs or symptoms secondary to mTBI at 1 to 3 months following a standardized information intervention provided in the acute setting. However, the pooled data failed to show an association between the intervention and the persistence of headache (relative risk $(R R)=0.88$; $95 \%$ 
Table 1 Characteristics of the studies

\section{First author, Inclusion criteria}

publication year

Studies including children only

Bell [34] Age $<16$ years old, $\mathrm{mTBl}$ of $<48$ hours' duration, $n=366$

Casey [29]

6 months to 14 years old. minor head trauma but exclusion of patients who loss consciousness

Ponsford [37] 6 to 15 years old, mTBI, GCS 13 to 15

Studies including adults and children

Af Geijerstam [41] > 5 years old, mTBI within the previous 24 hours, GCS of 15

Studies including mainly adults

Filipova [28]

18 to 60 years old mTB

Hinkle [30]

mTBl or skull fracture, GCS 13 to 15

Mittenberg [32]

Patients admitted for mTBI (adults), GCS 13 to 15

Paniak [31]

Adults, mTBI in the previous 3 weeks, exclusion of patients known to have psychiatric disorder

Number of Intervention

participants

Outcomes and results

Risk of bias ${ }^{\mathrm{a}}$ (specific areas of risk of bias)

366

Scheduled phone contact in the

first 3 months, standardized instruction handout, and a toll-free phone number CDC booklet (Facts about concussion and brain injury and where to get help), versus usual care

Discharge interview during which the nurse explained a take-home bookle of symptoms and phone follow-up carried out the day after discharge, versus usual care

Contacted in 48 hours and received neuropsychological assessment in 5 to 7 days plus information booklet, versus no follow-up and no booklet

Immediate CT scan of the head versus admission

Pharmaceutical intervention

Nasal DDAVP (10 $\mu$ g twice daily) for 5 days versus placebo

\section{Information at discharge}

Standardized information at discharge versus standardized information plus reassurance plus phone follow-up versus routine care

A 1 hour meeting with a therapist plus a 10 page manual plus a 10 minute questionnaire, versus routine care

Three to four hours of neuropsychological and personality assessment and treatment as needed plus single session with investigator session and a brochure, versus a
Fewer symptoms and less effect of

symptoms on functioning at 6 month

or the intervention group according

to the post-traumatic symptom

composite score (52.6 versus 46.0).

No difference in general health

composite score

No influence on a list of post-concussion symptoms 1 month after the accident

Potential (unclear for sequence allocation, concealment and blinding. No reporting of confounding and poor outcome measure)

Less post-concussion symptoms in the intervention group at 3 months

Potential (not randomized, no concealment confounding)

No statistically significant difference, Glasgow outcome scale not returned to normal at 3 months (21.4\% versus $242 \%$ )

Low

Intervention was associated with better results on information-processing test (PASAT) and verbal logical memory after 3 days of treatment. However, no effect seen on four other tests

Patient return to work and social activities in the information and information plus reassurance group occurred at least 1 week sooner than in the routine treatment group

Intervention associated with shorter duration of symptoms (33 versus 51 days) and fewer symptoms at follow-up at 6 months

No effect of intervention on social functioning and SF-36

Unclear (sequence allocation, concealment, blinding and outcome measure)

Unclear (sequence allocation, concealment blinding and confounding) 
Table 1 Characteristics of the studies (Continued)

\begin{tabular}{|c|c|c|c|c|c|}
\hline & & & $\begin{array}{l}\text { single session with investigator and } \\
\text { a brochure }\end{array}$ & & \\
\hline & & & Follow-up strategies & & \\
\hline Andersson $[33,42]$ & 16 to 60 years old, mTBI & 395 & $\begin{array}{l}\text { Telephone contact at } 2 \text { to } 8 \text { weeks, } \\
\text { follow-up in rehabilitation medicine, } \\
\text { and outpatient appointment weekly } \\
\text { as needed, versus usual care }\end{array}$ & $\begin{array}{l}\text { No difference in post-concussion } \\
\text { symptoms at } 1 \text { year or } 10 \text { years } \\
\text { after mTBl }\end{array}$ & Unclear (concealment) \\
\hline Ghaffar [35] & $\begin{array}{l}16 \text { to } 60 \text { years old, mTBl presenting } \\
\text { to the emergency department }\end{array}$ & 191 & $\begin{array}{l}\text { Follow-up in a multidisciplinary } \\
\text { clinic within } 1 \text { week and then as } \\
\text { needed, and treatment according } \\
\text { to specific complaints, versus no } \\
\text { follow-up }\end{array}$ & No effect on the RPCSQ & $\begin{array}{l}\text { Unclear (sequence allocation, concealment, } \\
\text { confounding, blinding, and fidelity to } \\
\text { protocol) }\end{array}$ \\
\hline Heskestad [36] & $\begin{array}{l}>15 \text { years old, minimal, mild and } \\
\text { moderate TBI }\end{array}$ & 326 & $\begin{array}{l}\text { Follow-up in neurosurgery clinic } \\
\text { within } 12 \text { to } 17 \text { days after the } \\
\text { accident, versus no follow-up }\end{array}$ & $\begin{array}{l}\text { No effect of intervention on post- } \\
\text { concussion symptoms }\end{array}$ & $\begin{array}{l}\text { Potential (not randomized. no concealment. } \\
15 \% \text { completed the study) }\end{array}$ \\
\hline Ponsford [39] & $>15$ years old, mTBl & 262 & $\begin{array}{l}\text { Contacted in } 48 \text { hours and received } \\
\text { neuropsychological assessment in } \\
5 \text { to } 7 \text { days plus information booklet, } \\
\text { versus no follow-up and no booklet }\end{array}$ & $\begin{array}{l}\text { Fewer post-concussion } \\
\text { symptoms related to anxiety } \\
\text { in the intervention group at } \\
3 \text { months }\end{array}$ & $\begin{array}{l}\text { Potential (not randomized, no concealment } \\
\text { confounding) }\end{array}$ \\
\hline \multirow[t]{2}{*}{ Wade [38] } & $\begin{array}{l}16 \text { to } 65 \text { years old, head injury of } \\
\text { any severity }\end{array}$ & 1156 & $\begin{array}{l}\text { Approached at } 7 \text { to } 10 \text { days after } \\
\text { injury and offered additional } \\
\text { information, advice, support, and } \\
\text { intervention as needed, versus no } \\
\text { follow-up }\end{array}$ & $\begin{array}{l}\text { No benefit on the RPCSQ at } \\
6 \text { months }\end{array}$ & Low \\
\hline & & & Other interventions & & \\
\hline De Kruijk [40] & $\begin{array}{l}>15 \text { years old, } \mathrm{mTBl} \text { of } 6 \text { hours } \\
\text { or more }\end{array}$ & 107 & $\begin{array}{l}\text { Full bed rest for } 6 \text { days followed } \\
\text { by gradual mobilization versus } \\
\text { gradual mobilization }\end{array}$ & $\begin{array}{l}\text { No effect of bed rest on } \\
\text { symptoms secondary to } \\
\text { concussion at } 2 \text { weeks, } 3 \\
\text { months, and } 6 \text { months }\end{array}$ & $\begin{array}{l}\text { Unclear (concealment, and fidelity to } \\
\text { protocol) }\end{array}$ \\
\hline Lowdon [19] & $\begin{array}{l}18 \text { to } 50 \text { years old, minor head } \\
\text { injury with loss of consciousness }\end{array}$ & 114 & $\begin{array}{l}\text { Admission overnight versus } \\
\text { discharge }\end{array}$ & $\begin{array}{l}\text { Admission had no effect on } \\
\text { the incidence and had a } \\
\text { deleterious effect on the } \\
\text { duration of symptoms for } \\
6 \text { weeks }\end{array}$ & $\begin{array}{l}\text { Unclear (sequence allocation, concealment, } \\
\text { and fidelity to protocol) }\end{array}$ \\
\hline
\end{tabular}

Test, RPCSQ Rivermead Post-Concussion Symptoms Questionnaire, SF-36 Short Form 36.

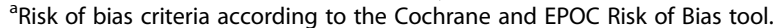




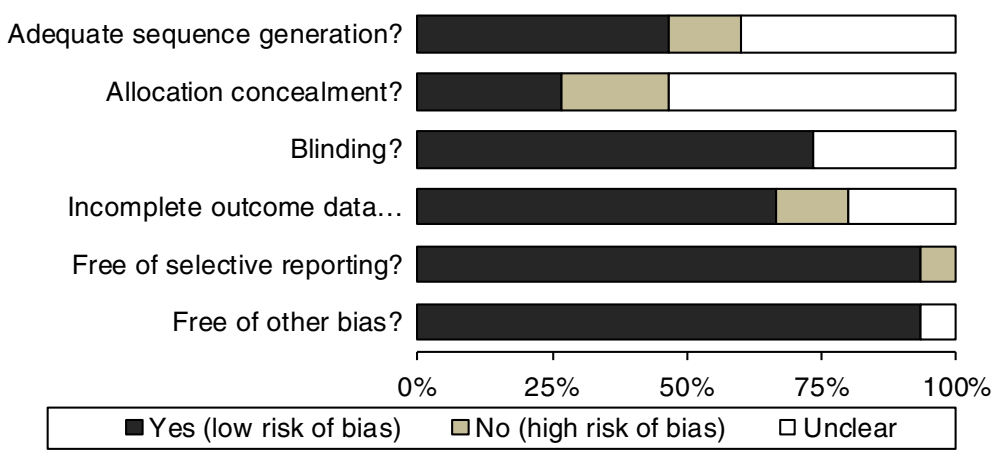

Figure 2 Summary of the risk of bias for the 15 studies.

CI 0.65 to 1.19 ) or vision impairment ( $R R=0.58$; $95 \%$ CI 0.10 to 3.31 ). The major limitations of this analysis related to the fact that the study population was different for the two articles (adults versus children) and that one of the studies had a potential risk of bias according to our evaluation. A funnel plot is provided in the supplementary material (Figure 3).

Seven studies evaluated the effect of a follow-up intervention compared with routine or no follow-up [33-39,42]. The interventions were of various types. For example, in one study, the intervention consisted of a phone follow-up, a neuropsychological evaluation at 1 week, and an information booklet [37], while in another study, the intervention was limited to a phone follow-up 2 to 8 weeks after the trauma [33]. Three studies showed a positive effect of the intervention [34,37,39]. These interventions were 1) an information booklet, phone follow-up in 48 hours, and follow-up in a specialized clinic 5 to 7 days after the trauma (evaluated in two studies); and 2) scheduled phone contact in the 3 months after trauma, in addition to an information handout and an information booklet. Importantly, two of these studies were potentially biased according to our evaluation. A meta-analysis was possible using four studies (one with children and three with adults) $[33,34,36,38]$ that reported outcomes on individual signs or symptoms secondary to mTBI at 6 to 12 months after trauma. No association was seen between the intervention and headache (RR 0.98; $95 \%$ CI 0.80 to 1.37 ), poor concentration (1.13; $95 \%$ CI 0.78 to 1.64$)$, memory problems (RR 1.17; 95\% CI 0.74 to 1.86), dizziness (RR 0.58 95\% CI 0.10 to 3.31 ), vision problems (RR 0.86; 95\% CI 0.65 to 1.14$)$, fatigue $(1.09 ; 95 \%$ CI 0.69 to 1.48$)$, irritability (1.03; $95 \%$ CI 0.79 to 1.35$)$, anxiety $(1.19 ; 95 \%$ CI 0.88 to $1.59)$, depression $(1.16 ; 95 \%$ CI 0.91 to 1.49$)$ or sensitivity to noise (1.17; $95 \%$ CI 0.74 to 1.86 ) (Figure 4a-j; see supplementary material). Many of these analyses identified important heterogeneity between studies with $\mathrm{I}^{2}$ values greater than $50 \%$. However, secondary analysis of the two studies [33,38] comparing follow-up intervention with no follow-up showed a positive effect for follow-up intervention

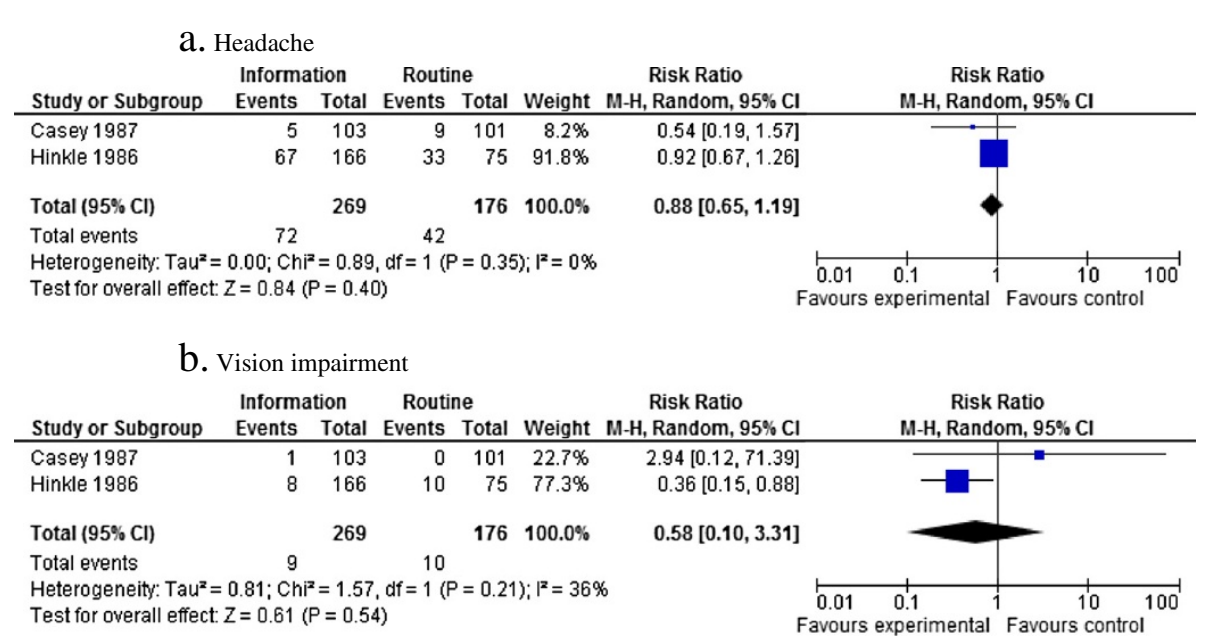

Figure 3 Association between standardized information interventions compared with routine or no information on multiple postconcussion symptoms at 1 to 3 months. 


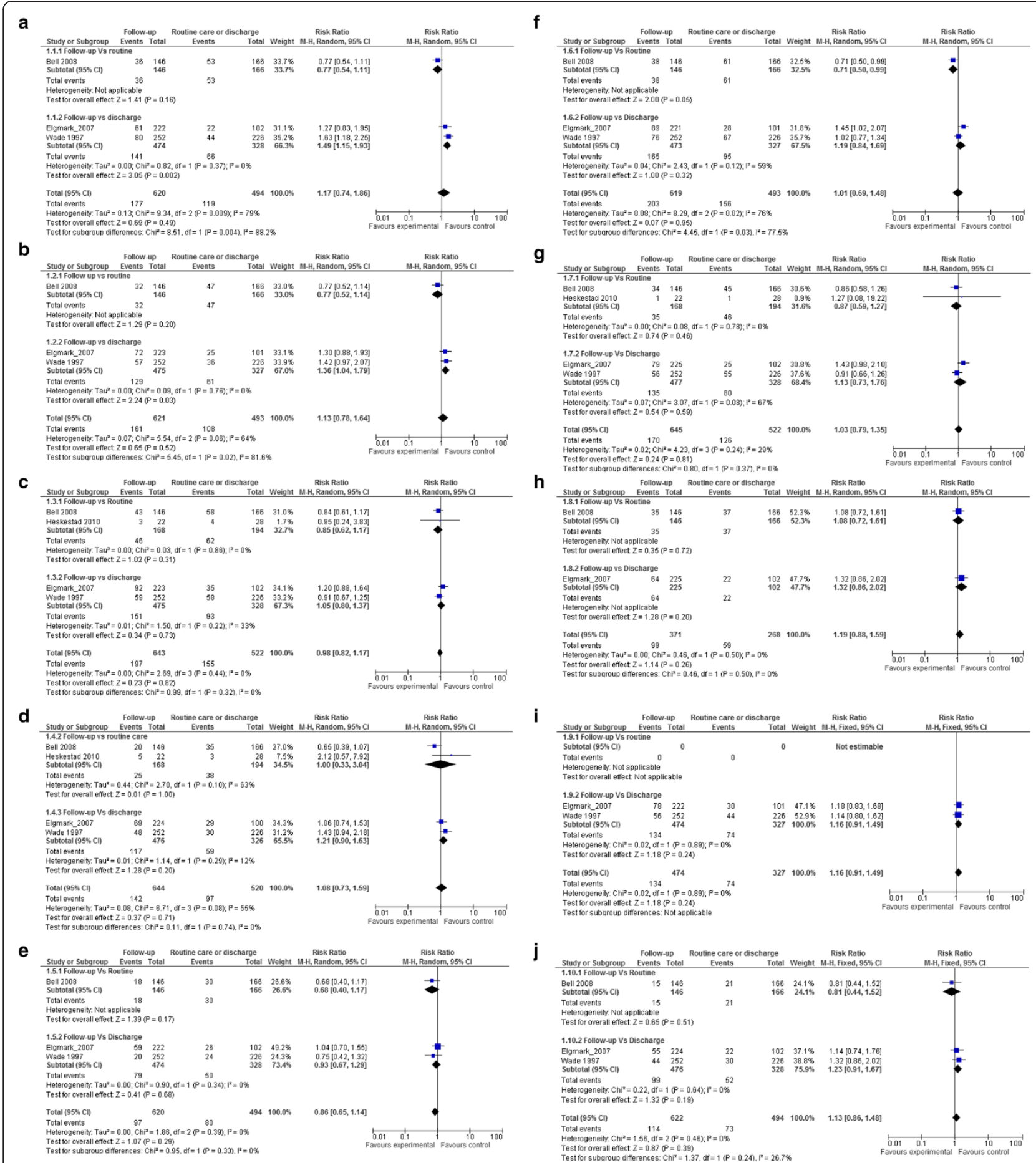

Figure 4 Association between follow-up interventions compared with routine or no follow-up on multiple post-concussion symptoms at 6-12 months. (a) Memory, (b) poor concentration, (c) headache, (d) dizziness, (e) vision impairment, (f) fatigue, (g) irritability, (h) anxiety, (i) depression, and (j) sensitivity to noise.

on the improvement of memory and concentration in adults (Figure 4a,b). Importantly, this sub-analysis showed no heterogeneity between studies.

One large study ( $\mathrm{n}=2523$ participants) failed to show any effect at 6 months for patients with mTBI who had an immediate head CT scan compared with admission [41]. Finally, two studies evaluating different strategies to restrict activity (full bed rest [40] or admission [19]) failed to show a clinical effect at 2 weeks to 6 months. 


\section{Variability in outcomes measured}

The reported primary outcomes were mostly related to post-concussion symptoms using various measurement tools (for example, Rivermead Post-Concussion Symptoms Questionnaire (RPCSQ) [43], visual analog scale (VAS), head injury checklist). Some studies reported data for individual symptoms. For example, presence of headache was assessed and reported in nine studies, and memory problems and dizziness were reported in seven studies. Other studies reported only a summary score for symptoms (for example, RPCSQ) or quality-of-life tools (for example, Short-Form Health survey [44], Glasgow Outcome Scale [45]). There was also important variability in the timeframe for outcome measurement. Only four studies reported outcomes measured in the first 2 months after mTBI, and five studies reported only outcomes occurring 6 months after intervention.

\section{Discussion}

This systematic review identified 15 randomized clinical trials evaluating an intervention provided or initiated in an acute setting for patients who sustained mTBI. Our study highlights the paucity of research regarding treatment for mTBI. Moreover, we found that a pharmacological intervention for patients who sustained mTBI has been evaluated in only one randomized clinical trial. This is particularly surprising considering that patients with mTBI represent more than $90 \%$ of all TBIs [21]. Our systematic review highlights the important heterogeneity in outcomes measured for patients who sustain mTBI. Although most studies reported many outcomes, no single primary outcome was clearly reported in the majority of studies.

Two systematic reviews of treatments for mTBI have been published previously. The WHO identified sixteen studies of interventions for patients who sustained mTBI [25], of which nine were randomized clinical trials and eight described an intervention that could be initiated at the ED. Three of the identified publications did not fulfill the inclusion criteria for our study: one trial was conducted before 1980 [46], another included only patients with persistent post-concussion symptoms at 3 months [47], and the third included all levels of TBI, from which it was impossible to isolate patients with mTBI [48]. The second systematic review, by Comper et al., identified twenty studies (including nine randomized clinical trials) of interventions for mTBI published between 1980 and 2003 [49]. Three of the randomized clinical trials failed our inclusion criteria because they included only patients with post-traumatic stress disorder [50] or headache 1 year after TBI [51], or because it was impossible to isolate only patients who had sustained a mTBI [48]. The conclusion of both reviews was that there was no high-quality intervention study pertaining to mTBI. In addition, their results suggested that a minimal educational strategy that also promotes return to activity as soon as possible might be effective. Our systematic review identified nine new studies not identified by the WHO review and eight not identified in the Compers et al. review. The main reason for this is because most of these studies were published after the completion of the previous reviews. Although our systematic review doubles the number of included studies, our conclusions are similar. In addition, it is interesting to note that no new randomized clinical trial on pharmacological treatment for $\mathrm{mTBI}$ has been reported for more than 20 years.

More recently, two systematic reviews focused on psychological treatments for mTBI [52,53]. The first review identified eight studies published between 2004 and 2006, in addition to ten studies described in a previous article [52]. Of these eighteen articles, twelve described a randomized clinical trial of which seven described an intervention provided in an acute setting for mTBI. These articles were all identified in our systematic review. This review concluded that there is little evidence to support any active treatment for mTBI but 'patient educational approaches may be beneficial if they are initiated in the early period following injury' [52]. The second review evaluated all psychological interventions for post-concussion symptoms published until November 2008. The authors identified seventeen randomized clinical trials, of which eight fulfilled our inclusion criteria and were included in our study. The authors concluded that there is some evidence of a positive effect of cognitive behavioral therapy for adults who sustain mTBI, whereas information, education or reassurance may not be as beneficial as previously thought' [53]. These results contrast with two systematic reviews providing evidence to support cognitive rehabilitation of patients who sustain stroke or TBI [54,55]. However, all patients included in the first systematic review had neurological deficits secondary to stroke or TBI [54], whereas the second study included only patients with moderate to severe TBI or patients with mTBI who consulted a rehabilitation clinic for persistent symptoms [55]. These patients do not reflect our study population, and this may explain the difference noted for the effects of cognitive rehabilitation.

The very small number of randomized clinical trials for patients who sustain mTBI is surprising, considering that these patients represent approximately $90 \%$ of all patients with TBI. This has an important influence on the research agenda regarding management of patients who sustain mTBI. Even though we identified only one pharmacological study for this study population, emergency physicians frequently prescribe medication for patients who have sustained mTBI [56]. There should be a rigorous evaluation of the mid-term and long-term effects of such medications on decreasing acute symptoms of mTBI. The 
possible reasons for the lack of publication of pharmacological studies are numerous. Publication bias is not likely to be a factor because no pertinent study was identified by our group in the conference proceedings of five relevant conferences. Rather, there is probably a lack of proofof-concept research on pharmacological intervention. However, it will be difficult to have an animal model for post-concussion syndrome. Accordingly, clinical researchers should not wait for animal data before suggesting clinical trials for humans who sustain mTBI. Initial studies could evaluate the effects at 1 week of symptom-driven treatment for mTBI. Most guidelines suggest activity restriction after mTBI $[57,58]$, but our systematic review failed to find a positive effect for this type of intervention, and further study is needed in this area.

Our study highlights the wide variety of clinical outcomes reported in the included studies. Furthermore, studies used different measurement tools for the same outcome. For example, post-concussion symptoms were reported individually (for example,: presence or absence of headache) or using a composite score grouping many symptoms (for example,: Rivermead Post-Concussion Symptoms Questionnaire (RPCSQ, VAS for each symptom, head injury questionnaire). Variability was also related to the timing of measurement. While one study evaluated the effect of the intervention at 3 days, others measured it at 6 to 12 months. Variability in outcome may be related to the presence of divergent definitions of persistent post-concussion syndrome. For example, persistent postconcussion syndrome occurs after 3 months according to the DSM-IV-TR [59], but after only 1 month according to the ICD-10 [60]. This variability has been evoked in the past, and authors have suggested standardization [61-63]. Moreover, the $\mathrm{NIH}$, through the National Institute of Neurological Disorders and Stroke (NINDS), developed the Common Data Element (CDE) project (http://www. commondataelements.ninds.nih.gov/\#page=Default). The goal of this initiative was to develop data standards for neurology clinical research. The initiative permitted the creation of common definitions and datasets to consistently capture and record outcomes across clinical studies in neurology. In our systematic review, the outcomes most commonly reported were the presence or absence of headache, dizziness, or memory problems.

\section{Limitations}

The most important limitation to our systematic review is related to the large heterogeneity of interventions and outcomes. This limited our ability to group studies together based on intervention. In addition, the wide diversity in outcome measures and timing of their measurement limited our ability to conduct a meta-analysis including most studies. However, this in turn is one of the main findings of this study, further supporting the need to standardize definitions and methods in order to advance research in the field. A qualitative description of the interventions and the studies will help readers come to a better understanding of the subject. As with all systematic reviews, there is an inherent limitation related to publication bias. The small number of studies and their heterogeneity limited our ability to evaluate the extent of publication bias.

\section{Conclusion}

Our systematic review demonstrates a paucity of clinical trials for patients who sustain mTBI. According to the published literature, no intervention initiated acutely has been clearly associated with a positive outcome for patients who sustain $\mathrm{mTBI}$, and there is little evidence suggesting that follow-up interventions may be associated with a better outcome. Considering the high proportion of patients with persistent symptoms after mTBI, it would seem essential to evaluate potential treatments to decrease these symptoms.

Finally, the large spectrum of outcomes evaluated for patients who sustain mTBI limits the possibilities for comparing treatments. Although a composite index score (continuous data) comprising many symptoms secondary to mTBI may be more useful for research purposes, researchers should also collect data on individual symptoms to permit comparison of studies. In addition, researchers should measure outcomes at 1 week, 1 month and 3 months after trauma. One potential research idea may be to ask patients having sustained a mTBI what outcomes would be meaningful for them.

\section{Abbreviations}

CDC: Centers for Disease Control; CT: computed tomography; DDAVP: 1-deamino-8-D-arginine vasopressin; DSM-IV-TR: Diagnostic and Statistical Manual of Mental Disorders, fourth edition, tenth revision; ED: Emergency department; GCS: Glasgow Coma Scale; ICD-10: International Statistical Classification of Disease and Related Health Problems, 10th edition; ITT: Intention-to-treat; mTBI: Mild traumatic brain injury; PASAT: Paced Auditory Serial Addition Test; RPCSQ: Rivermead Post-Concussion Symptoms Questionnaire; SF-36: Short Form 36; VAS: visual analog scale; WHO: World Health Organization.

\section{Competing interests}

The authors declare that they have no competing interests.

\section{Authors' contributions}

JG conceived the study and obtained funding. All collaborators contributed to the design. $A D A, B C, J G$, and $M W$ participated in the study selection and evaluated the articles. MB, JMC, LC, and JG performed the manual search of the conference proceedings. NC and MW performed the primary analysis of the data. JG drafted the manuscript, and all authors contributed substantially to its revision. JG takes responsibility for the paper as a whole. Each author listed on the manuscript has seen and approved the submission of this version of the manuscript and takes full responsibility for the manuscript.

\section{Acknowledgements}

The study was financially supported by the Fonds de Recherche du QuébecSanté (FRQ-S). Grant number: 26219. The funding agency had no role in the study design, in the collection, analysis and interpretation of the data, or in the writing of and the decision to submit the paper. 


\section{Author details}

'Département de Pédiatrie, CHU Sainte-Justine, Université de Montréal, Montréal, Canada. ²Département de Chirurgie, CHU Sainte-Justine, Université de Montréal, Montréal, Canada. ${ }^{3}$ Département de psychologie, Université de Montréal, Montréal, Canada. ${ }^{4}$ Département d’urgence, Hôpital Sacré-Cœur, Université de Montréal, Montréal, Canada. ${ }^{5}$ Centre de recherche du CHU Sainte-Justine, Université de Montréal, Montréal, Canada. ${ }^{6}$ Section d'Urgence, Département de Pédiatrie, CHU Sainte-Justine, 3175 Chemin Côte SainteCatherine, Montréal QC H3T 1C5, Canada.

Received: 15 March 2013 Accepted: 30 May 2013

Published: 7 August 2013

\section{References}

1. Ruff RM, Iverson GL, Barth JT, Bush SS, Broshek DK: Recommendations for diagnosing a mild traumatic brain injury: a National Academy of Neuropsychology education paper. Arch Clin Neuropsychol 2009, 24:3-10.

2. Mild Traumatic Brain Injury Committee of the Head Injury Interdisciplinary Special Interest Group of the American Congress of Rehabilitation Medicine: Definition of mild traumatic brain injury. J Head Trauma Rehabil 1993, 8:68-69.

3. Cassidy JD, Carroll L, Peloso PM, Borg J, von Hoist H, Holm L, Kraus J, Coronado VG: Incidence, risk factors and prevention of mild traumatic brain injury: results of the WHO Collaborating Centre Task Force on Mild Traumatic Brain Injury. J Rehabil Med 2004, 43:28-60.

4. Cassidy JD, Carroll L, Cote P, Holm L, Nygren A: Mild traumatic brain injury after traffic collisions: a population-based inception cohort study. J Rehabil Med 2004, 43:15-21.

5. Sosin DM, Sniezek JE, Thurman DJ: Incidence of mild and moderate brain injury in the United States, 1991. Brain Inj 1996, 10:47-54.

6. Boden BP, Kirkendall DT, Garrett WE Jr: Concussion incidence in elite college soccer players. Am J Sports Med 1998, 26:238-241.

7. Powell JW, Barber-Foss KD: Traumatic brain injury in high school athletes. JAMA 1999, 282:958-963.

8. Buckley WE: Concussions in college football. A multivariate analysis. Am J Sports Med 1988, 16:51-56.

9. Jorgensen U, Schmidt-Olsen S: The epidemiology of ice hockey injuries. Br J Sports Med 1986, 20:7-9.

10. Tegner $Y$, Lorentzon R: Concussion among Swedish elite ice hockey players. Br J Sports Med 1996, 30:251-255.

11. Roberts WO, Brust JD, Leonard B: Youth ice hockey tournament injuries: rates and patterns compared to season play. Med Sci Sports Exerc 1999, 31:46-51.

12. Rivara FP: Childhood injuries. III: Epidemiology of non-motor vehicle head trauma. Dev Med Child Neurol 1984, 26:81-87.

13. Rivara FP, Bergman AB, LoGerfo JP, Weiss NS: Epidemiology of childhood injuries. II. Sex differences in injury rates. Am J Dis Child 1982, 136:502-506.

14. Durkin MS, Olsen S, Barlow B, Virella A, Connolly ES Jr: The epidemiology of urban pediatric neurological trauma: evaluation of, and implications for, injury prevention programs. Neurosurgery 1998, 42:300-310.

15. Carroll LJ, Cassidy JD, Peloso PM, Borg J, von Holst H, Holm L, Paniak C, Pepin M: Prognosis for mild traumatic brain injury: results of the WHO Collaborating Centre Task Force on Mild Traumatic Brain Injury. J Rehabil Med 2004, 43:84-105.

16. King NS, Kirwilliam S: Permanent post-concussion symptoms after mild head injury. Brain Inj 2011, 25:462-470.

17. King NS, Crawford S, Wenden FJ, Caldwell FE, Wade DT: Early prediction of persisting post-concussion symptoms following mild and moderate head injuries. Br J Clin Psychol 1999, 38(Pt 1):15-25

18. King NS: Emotional, neuropsychological, and organic factors: their use in the prediction of persisting postconcussion symptoms after moderate and mild head injuries. J Neurol Neurosurg Psychiatry 1996, 61:75-81.

19. Lowdon IM, Briggs M, Cockin J: Post-concussional symptoms following minor head injury. Injury 1989, 20:193-194.

20. Cushman JG, Agarwal N, Fabian TC, Garcia V, Nagy KK, Pasquale MD, Salotto AG: Practice management guidelines for the management of mild traumatic brain injury: the EAST practice management guidelines work group. J Trauma 2001, 51:1016-1026.

21. McCrory P, Meeuwisse W, Johnston K, Dvorak J, Aubry M, Molloy M, Cantu $\mathrm{R}$ : Consensus statement on concussion in sport: the 3rd International Conference on Concussion in Sport held in Zurich, November 2008. Br J Sports Med 2009, 43(Suppl 1):i76-i90.
22. Standaert CJ, Herring SA, Cantu RC: Expert opinion and controversies in sports and musculoskeletal medicine: concussion in the young athlete. Arch Phys Med Rehabil 2007, 88:1077-1079.

23. Willer B, Leddy JJ: Management of concussion and post-concussion syndrome. Curr Treat Options Neurol 2006, 8:415-426.

24. Borg J, Holm L, Cassidy JD, Peloso PM, Carroll LJ, von Hoist H, Ericson K: Diagnostic procedures in mild traumatic brain injury: results of the WHO Collaborating Centre Task Force on Mild Traumatic Brain Injury. J Rehabil Med 2004, 43:61-75.

25. Borg J, Holm L, Peloso PM, Cassidy JD, Carroll LJ, von Hoist H, Paniak C, Yates D: Non-surgical intervention and cost for mild traumatic brain injury: results of the WHO Collaborating Centre Task Force on Mild Traumatic Brain Injury. J Rehabil Med 2004, 43:76-83.

26. Higgins JPT GeS: Cochrane Handbook for Systematic Reviews of Interventions Version 5.0.2. In The Cochrane Library, Issue 5, 2008. Chichester, UK: John Wiley \& Sons, Ltd; 2008.

27. Hartling L, Ospina M, Liang Y, Dryden DM, Hooton N, Krebs SJ, Klassen TP. Risk of bias versus quality assessment of randomised controlled trials: cross sectional study. BMJ 2009, 339:b4012.

28. Filipova M, Jung M, Filip M, Krejcova H: Clinical efficacy of 1-desamino-8-darginine-vasopressin (DDAVP) in short-term recovery from minor head injury. Hum Psychopharmacol 1989, 4:47-50

29. Casey R, Ludwig S, McCormick MC: Minor head trauma in children: an intervention to decrease functional morbidity. Pediatrics 1987, 80:159-164.

30. Hinkle $\mathrm{J}$, Alves WM, Rimell RW, Jane JA: Restoring social competence in minor head-injury patients. J Neurosci Nurs 1986, 18:268-271.

31. Paniak C, Toller-Lobe G, Durand A, Nagy J: A randomized trial of two treatments for mild traumatic brain injury. Brain Inj 1998, 12:1011-1023.

32. Mittenberg W, Tremont G, Zielinski RE, Fichera S, Rayls KR: Cognitivebehavioral prevention of postconcussion syndrome. Arch Clin Neuropsychol 1996, 11:139-145

33. Elgmark AE, Emanuelson I, Bjorklund R, Stalhammar DA: Mild traumatic brain injuries: the impact of early intervention on late sequelae. A randomized controlled trial. Acta Neurochir (Wien) 2007, 149:151-159.

34. Bell KR, Hoffman JM, Temkin NR, Powell JM, Fraser RT, Esselman PC, Barber JK, Dikmen S: The effect of telephone counselling on reducing posttraumatic symptoms after mild traumatic brain injury: a randomised trial. J Neurol Neurosurg Psychiatry 2008, 79:1275-1281.

35. Ghaffar O, McCullagh S, Ouchterlony D, Feinstein A: Randomized treatment trial in mild traumatic brain injury. J Psychosom Res 2006, 61:153-160.

36. Heskestad B, Waterloo K, Baardsen R, Helseth E, Romner B, Ingebrigtsen T: No impact of early intervention on late outcome after minimal, mild and moderate head injury. Scand J Trauma Resusc Emerg Med 2010, 18:10.

37. Ponsford J, Willmott C, Rothwell A, Cameron P, Ayton G, Nelms R, Curran C, $\mathrm{Ng} \mathrm{K}$ : Impact of early intervention on outcome after mild traumatic brain injury in children. Pediatrics 2001, 108:1297-1303.

38. Wade DT, Crawford S, Wenden FJ, King NS, Moss NE: Does routine follow up after head injury help? A randomised controlled trial. $J$ Neurol Neurosurg Psychiatry 1997, 62:478-484.

39. Ponsford J, Willmott C, Rothwell A, Cameron P, Kelly AM, Nelms R, Curran C: Impact of early intervention on outcome following mild head injury in adults. J Neurol Neurosurg Psychiatry 2002, 73:330-332.

40. de Kruijk JR, Leffers P, Meerhoff S, Rutten J, Twijnstra A: Effectiveness of bed rest after mild traumatic brain injury: a randomised trial of no versus six days of bed rest. J Neurol Neurosurg Psychiatry 2002, 73:167-172.

41. af Geijerstam JL, Oredsson S, Britton M: Medical outcome after immediate computed tomography or admission for observation in patients with mild head injury: randomised controlled trial. BMJ 2006, 333:465.

42. Andersson EE, Bedics BK, Falkmer T: Mild traumatic brain injuries: a 10year follow-up. J Rehabil Med 2011, 43:323-329.

43. King NS, Crawford S, Wenden FJ, Moss NE, Wade DT: The Rivermead Post Concussion Symptoms Questionnaire: a measure of symptoms commonly experienced after head injury and its reliability. J Neurol 1995, 242:587-592.

44. Ware JE Jr, Sherbourne CD: The MOS 36-item short-form health survey (SF-36). I. Conceptual framework and item selection. Med Care 1992, 30:473-483.

45. Pettigrew LE, Wilson JT, Teasdale GM: Reliability of ratings on the Glasgow Outcome Scales from in-person and telephone structured interviews. J Head Trauma Rehabil 2003, 18:252-258.

46. Relander M, Troupp H, Af BG: Controlled trial of treatment for cerebral concussion. Br Med J 1972, 4:777-779. 
47. Chapman EH, Weintraub RJ, Milburn MA, Pirozzi TO, Woo E: Homeopathic treatment of mild traumatic brain injury: a randomized, double-blind, placebo-controlled clinical trial. J Head Trauma Rehabil 1999, 14:521-542.

48. Wade DT, King NS, Wenden FJ, Crawford S, Caldwell FE: Routine follow up after head injury: a second randomised controlled trial. J Neurol Neurosurg Psychiatry 1998, 65:177-183.

49. Comper P, Bisschop SM, Carnide N, Tricco A: A systematic review of treatments for mild traumatic brain injury. Brain Inj 2005, 19:863-880.

50. Bryant RA, Moulds M, Guthrie R, Nixon RD: Treating acute stress disorder following mild traumatic brain injury. Am J Psychiatry 2003, 160:585-587.

51. Jensen OK, Nielsen FF, Vosmar L: An open study comparing manual therapy with the use of cold packs in the treatment of post-traumatic headache. Cephalalgia 1990, 10:241-250.

52. Snell DL, Surgenor $L$, Hay-Smith EJ, Siegert RJ: A systematic review of psychological treatments for mild traumatic brain injury: an update on the evidence. J Clin Exp Neuropsychol 2009, 31:20-38.

53. Al SA, Sandford D, Carson AJ: Psychological approaches to treatment of postconcussion syndrome: a systematic review. J Neurol Neurosurg Psychiatry 2010, 81:1128-1134.

54. Cicerone KD, Langenbahn DM, Braden C, Malec JF, Kalmar K, Fraas M, Felicetti T, Laatsch L, Harley JP, Bergquist T, Azulay J, Cantor J, Ashman T: Evidence-based cognitive rehabilitation: updated review of the literature from 2003 through 2008. Arch Phys Med Rehabil 2011, 92:519-530.

55. Carney N, Chesnut RM, Maynard H, Mann NC, Patterson P, Helfand M: Effect of cognitive rehabilitation on outcomes for persons with traumatic brain injury: A systematic review. J Head Trauma Rehabil 1999, 14:277-307.

56. Sturm JJ, Simon HK, Hirsh DA: The use of ondansetron for nausea and vomiting after head injury and its effect on return rates from the pediatric ED. Khan NS: Am J Emerg Med; 2012.

57. Carroll LJ, Cassidy JD, Holm L, Kraus J, Coronado VG: Methodological issues and research recommendations for mild traumatic brain injury: the WHO Collaborating Centre Task Force on Mild Traumatic Brain Injury. J Rehabil Med 2004:113-125.

58. Marshall S, Bayley M, McCullagh S, Velikonja D, Berrigan L: Clinical practice guidelines for mild traumatic brain injury and persistent symptoms. Can Fam Physician 2012, 58:257-267.

59. American Psychiatric Association: Diagnostic and Statistical Manual of Mental Disorders. Washington, DC: American Psychiatric Association; 2000.

60. WHO: International Statistical Classification of Disease and Related Health Problems. Geneva, Switzerland: World Health Organization; 1992.

61. Lee LK: Controversies in the sequelae of pediatric mild traumatic brain injury. Pediatr Emerg Care 2007, 23:580-583.

62. Ruff RM: Mild traumatic brain injury and neural recovery: rethinking the debate. NeuroRehabilitation 2011, 28:167-180.

63. Wilde EA, Whiteneck GG, Bogner J, Bushnik T, Cifu DX, Dikmen S, French L, Giacino JT, Hart T, Malec JF, Millis SR, Novack TA, Sherer M, Tulsky DS, Vanderploeg RD, von Steinbuechel N: Recommendations for the use of common outcome measures in traumatic brain injury research. Arch Phys Med Rehabil 2010, 91:1650-1660.

doi:10.1186/2046-4053-2-63

Cite this article as: Gravel et al:: Interventions provided in the acute phase for mild traumatic brain injury: a systematic review. Systematic Reviews 2013 2:63.

\section{Submit your next manuscript to BioMed Central and take full advantage of:}

- Convenient online submission

- Thorough peer review

- No space constraints or color figure charges

- Immediate publication on acceptance

- Inclusion in PubMed, CAS, Scopus and Google Scholar

- Research which is freely available for redistribution

Submit your manuscript at www.biomedcentral.com/submit
( Biomed Central 